\title{
Interdigital cantilevers for atomic force microscopy
}

\author{
S. R. Manalis, S. C. Minne, A. Atalar, ${ }^{a)}$ and C. F. Quate \\ E. L. Ginzton Laboratory, Stanford University, California 94305-4085
}

(Received 9 September 1996; accepted for publication 2 October 1996)

\begin{abstract}
We present a sensor for the atomic force microscope (AFM) where a silicon cantilever is micromachined into the shape of interdigitated fingers that form a diffraction grating. When detecting a force, alternating fingers are displaced while remaining fingers are held fixed. This creates a phase sensitive diffraction grating, allowing the cantilever displacement to be determined by measuring the intensity of diffracted modes. This cantilever can be used with a standard AFM without modification while achieving the sensitivity of the interferometer and maintaining the simplicity of the optical lever. Since optical interference occurs between alternating fingers that are fabricated on the cantilever, laser intensity rather than position can be measured by crudely positioning a photodiode. We estimate that the rms noise of this sensor in a $10 \mathrm{hz}-1 \mathrm{kHz}$ bandwidth is $\sim 0.02 \AA$ and present images of graphite with atomic resolution. (C) 1996 American Institute of Physics. [S0003-6951(96)00851-0]
\end{abstract}

The cantilever with an integrated tip is a key component in the family of microscopes known as the scanning probes. Its importance stems from its crucial role in determining the sensitivity of the system. Atomic force microscopes (AFM) commonly measure deflections that are much less than $1 \AA$ using techniques such as tunneling, ${ }^{1}$ optical lever detection, ${ }^{2,3}$ interferometry, ${ }^{4-7}$ and piezoresistive sensing. ${ }^{8}$ This has allowed the structure of a variety of crystalline surfaces to be imaged on the atomic level. High-resolution imaging is particularly useful in applications such as subangstrom surface roughness measurements and profiling the structure of DNA.

One of the most sensitive optical techniques for measuring the deflection of a cantilever is the interferometer. Rugar et $a{ }^{6}{ }^{6}$ developed a deflection sensor based on the interference of light between the cleaved end of an optical fiber and the backside of a cantilever. By accurately positioning the fiber above the cantilever to form a tightly spaced interference cavity of less than $4 \mu \mathrm{m}$, it is possible to achieve a vertical resolution on the order of $0.01 \AA$. In other work, Schönenberger and Alvarado ${ }^{7}$ developed a scheme where a birefringent prism is used to divide a laser into a sensing and reference beam. The prism is mounted within a few millimeters of the cantilever such that the reference beam is reflected off the cantilever base while the sensing beam is reflected near the tip. The back-reflected light is then analyzed with an additional birefringent prism and directed to a split photodiode. Such a system reduced the perturbations resulting from fluctuations of the optical path length and also yielded a resolution of $\sim 0.01 \AA$.

The process of optically measuring deflection can be simplified through a technique known as the optical lever. In this system, a laser beam is reflected off the backside of the cantilever and directed into a split photodiode. The position of the reflected beam and, hence, the cantilever deflection, is determined by subtracting the photodiode outputs. Unlike the interferometer, the optical lever does not require the positioning of components directly above the cantilever. It is this

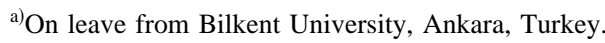

simplicity that has made the optical lever more popular than the interferometer. However, the resolution is typically limited to roughly $0.1 \AA{ }^{9}$

In this letter, we present a new interferometric sensor for the AFM where a cantilever is micromachined into the shape of interdigitated fingers to form a diffraction grating. Operationally, this technique requires an illumination source and a standard photodiode, yet it achieves a resolution that is comparable to the interferometric sensors described previously. The improved simplicity allows the interdigital cantilever to be used in most optical lever AFMs without modification. It should also be useful in a number of applications where the cantilever is used to observe physical and chemical events. ${ }^{9,10}$

Micromachined diffraction grating are used in many micro-optical systems ${ }^{11}$ and can be used for high-resolution displays. ${ }^{12}$ The idea of integrating a diffraction grating onto the cantilever to determine its deflection was suggested by one of us (A.A.) while searching for a high-resolution deflection sensor for arrays of cantilevers. The interdigital cantilever alleviates the task of critically aligning an array of photodiodes since intensity rather than position of the reflected beam is measured.

A scanning electron micrograph (SEM) of an interdigital cantilever is shown in Fig. 1. The cantilever is defined such that when a force acts on the tip, only the alternating fingers that are connected to the outer portion of the cantilever are vertically displaced. The remaining set of fingers, or reference fingers, are attached to the inner portion of the cantilever and remain fixed. When the cantilever is illuminated, the fingers form a phase sensitive diffraction grating, and the tip displacement is determined by measuring the intensity of the diffracted modes. The dominant reflected mode from the grating when the cantilever is not deflected is the zeroth mode. As the tip is displaced by an external force, the interference between the light reflecting off the reference fingers and the moving fingers causes the zeroth mode intensity to decrease while a first mode is created. ${ }^{13}$ When the cantilever is deflected by an amount of $\lambda / 4$, where $\lambda$ is the wavelength of the illumination source, the zeroth mode is minimized and the first mode is maximized. The cantilever deflection can be 


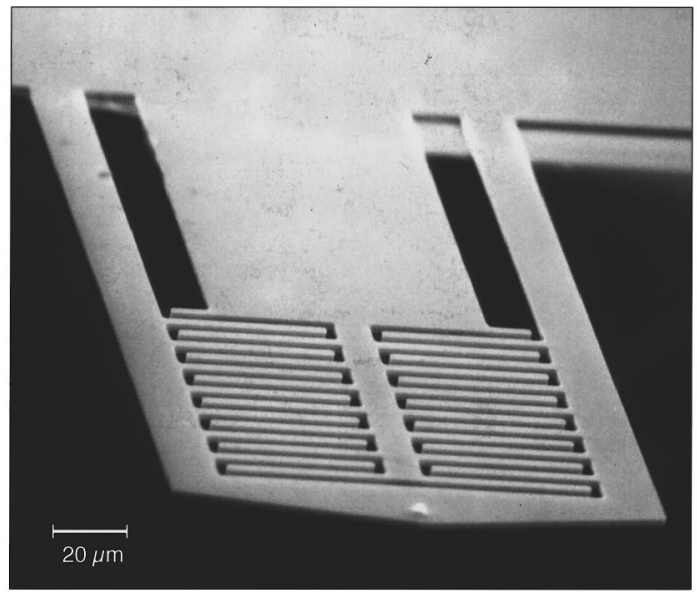

FIG. 1. SEM micrograph of an interdigital cantilever that is $215 \mu \mathrm{m}$ long, $2.5 \mu \mathrm{m}$ thick, and contains $3 \mu \mathrm{m}$ wide fingers.

determined by measuring the intensity of the zeroth mode, first mode, or the difference between the modes. Maximum sensitivity of the deflection occurs at $\lambda / 8$. The distance between the zeroth and first mode is roughly $2 \mathrm{~mm}$ for our system.

The fabrication of the interdigital cantilever is a three mask process that begins by growing $1 \mu \mathrm{m}$ of thermal oxide on an a $<100>$ silicon-on-insulator wafer on which the top silicon is $10 \mu \mathrm{m}$ of undoped epitaxial silicon. Tip masks are patterned into the oxide with 6:1 HF, undercut into the epitaxial silicon with a plasma etch, and sharpened by a wet oxidation at $950{ }^{\circ} \mathrm{C}$ for $2 \mathrm{~h}$. The cantilever and the interdigitated fingers are defined in a plasma etch. The top surface is then passivated with polyimide and the bulk silicon is etched with ethylene diamine pyrocathecol using the middle oxide as an etch stop. Cantilevers are released by etching the middle oxide in 6:1 $\mathrm{HF}$ and removing the polyimide in an oxygen plasma (see Fig. 1). All measurements and images obtained with the interdigital cantilever use a commercial microscope head ${ }^{14}$ with a homebuilt scanning system and control electronics.

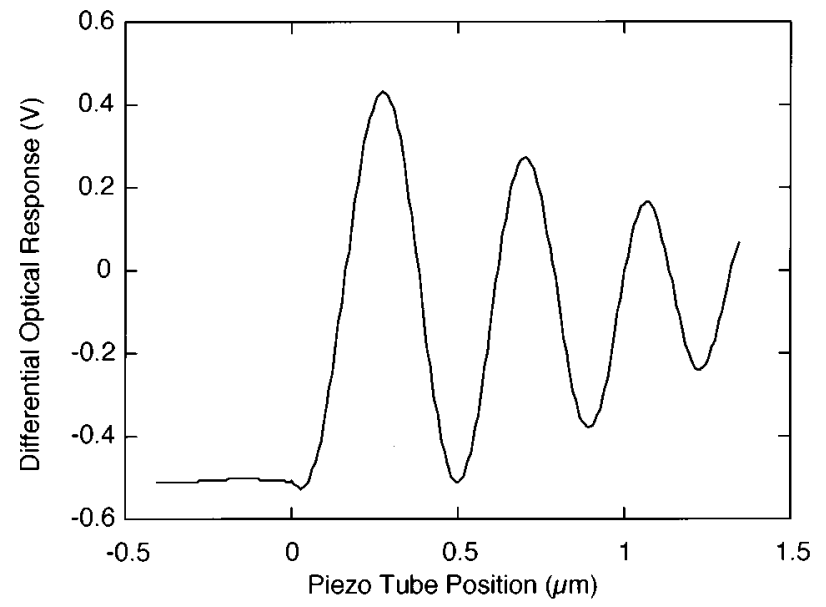

FIG. 2. Force curve obtained by recording the intensity difference between the zeroth and first modes when the cantilever is deflected by displacing the tip with a piezotube. The amplitude of the optical response decays since the vertical separation between the moving and reference fingers is not constant due to the curvature of the deflected cantilever.

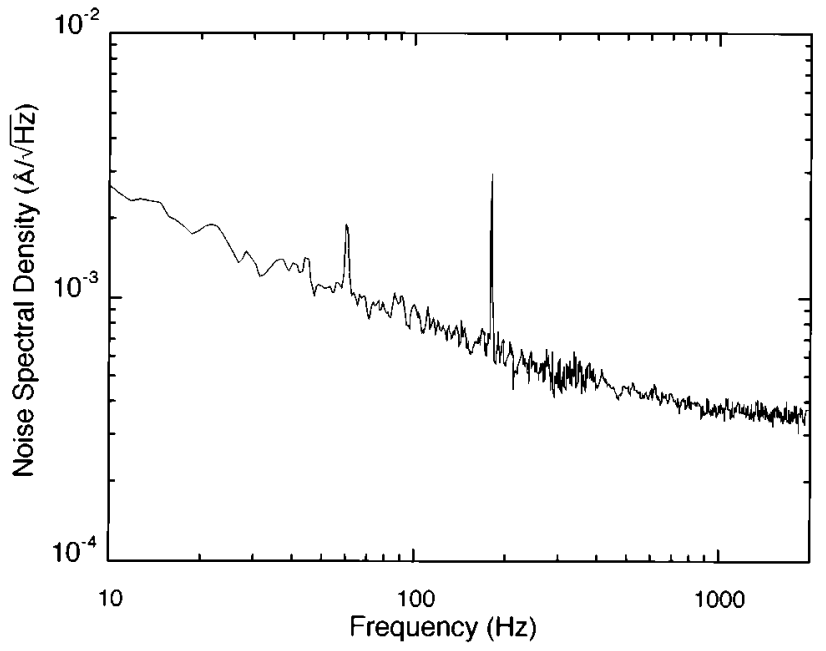

FIG. 3. Noise spectral density measured when the tip end of the cantilever is free. The maximum slope from the force curve shown in Fig. 2 was used to calibrate the noise spectral density.

Figure 2 shows a typical force curve that is obtained by measuring the intensity difference between the zeroth and first modes as a function of cantilever deflection. The cantilever is deflected by applying a force at the tip with a piezotube. A laser diode with a wavelength of $670 \mathrm{~nm}$ is focused to a $\sim 20 \mu \mathrm{m}$ spot and aligned to a set of interdigitated fingers. The spot is placed such that the longitudinal finger support is not illuminated. The intensity of the diffracted modes is measured with a split photodiode placed roughly 4 $\mathrm{cm}$ from the cantilever. Note that the period of the optical response shown in Fig. 3(a) is $470 \mathrm{~nm}$, which is slightly larger than the expected value of $\lambda / 2=335 \mathrm{~nm}$. We find that this discrepancy can be accounted for by considering the following effects: Since the force is applied at the tip and the laser beam is focused on the diffraction grating, the actual vertical finger displacement is less than the tip deflection due to the bending of the cantilever. Also, the expected period is increased because the laser beam does not reflect off the diffraction grating with normal incidence.

Resolution of the interdigital cantilever is estimated by measuring the noise spectral density of a free-standing cantilever (see Fig. 3). To calibrate this measurement in terms of position, the noise spectral density is divided by the cantilever sensitivity, which is the maximum slope of the force curve shown in Fig. 2. This yields a rms noise of roughly $0.02 \AA$ in a $10 \mathrm{~Hz}$ to $1 \mathrm{kHz}$ bandwidth. However, the noise spectral density is measured at a point on the force curve where the sensitivity is not maximized. In order to understand the meaning of this measurement, the following issues should be considered. First, the noise spectral density does not include an accurate measure of mechanical noise of the cantilever. Theoretically, we estimate that the thermal mechanical noise of the cantilever is $\sim 0.005 \AA$ in a $1 \mathrm{kHz}$ bandwidth. ${ }^{15,16}$ Second, the combined laser power from the zeroth and first modes incident on the split photodiode is constant with respect to cantilever deflection and corresponds to a shot noise of $0.007 \AA .{ }^{17}$ Since the contribution of thermal mechanical and shot noise is small compared to our noise measurement of $0.02 \AA$, we speculate that a significant noise source is intensity fluctuations of the laser. In our mea- 
(a)

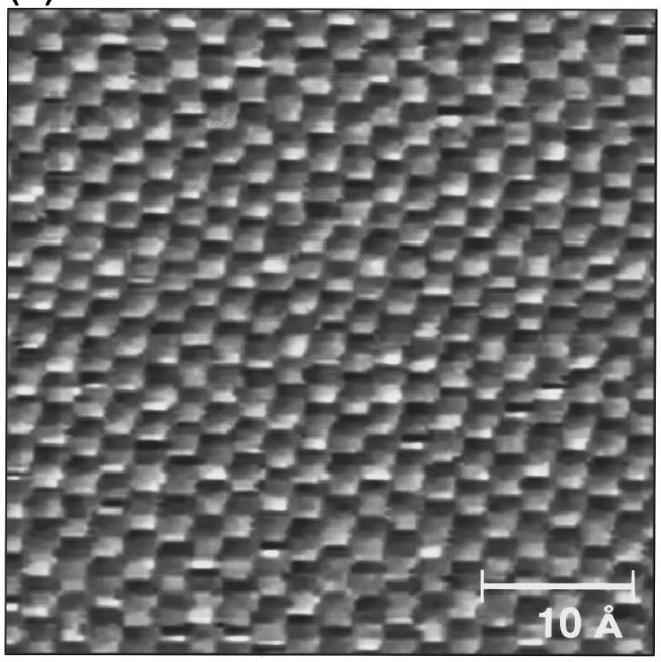

(b)

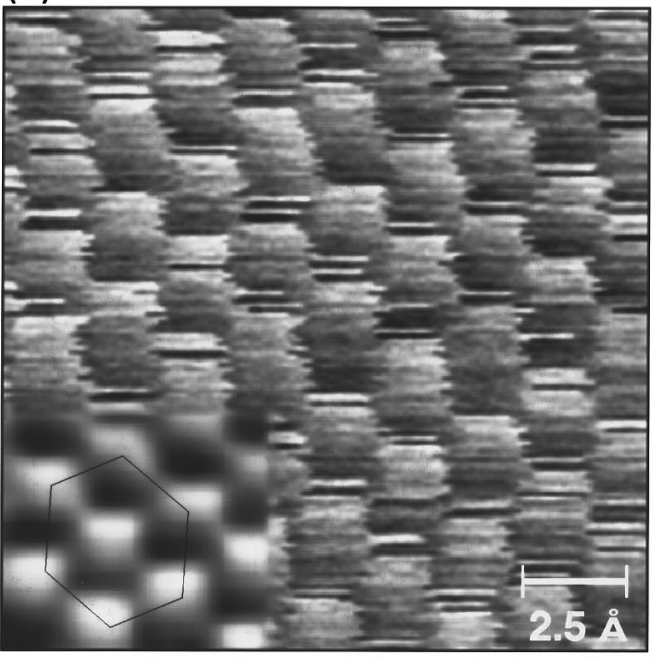

FIG. 4. (a) $35 \AA \times 35 \AA$ atomic image of graphite imaged by the interdigital cantilever. (b) Image area is reduced to $20 \AA \times 20 \AA$. The data in the bottom left-hand corner has been filtered to clearly show the hexagonal structure of graphite.

surement, the zeroth order intensity is maximized and the first order intensity is minimized since the cantilever is not deflected. As a result, laser intensity noise is not correctly subtracted through the differential output. This implies that the measured noise of $0.02 \AA$ may be an upper limit that could be reduced if laser intensity noise can be canceled by subtraction.

When imaging, the tip is placed into contact with a sample and the vertical position of the piezotube is adjusted to maximize the cantilever sensitivity. Atomic images of graphite are shown in Fig. 4. The data in Fig. 4(b) is raw with the exception of the lower left-hand corner, which has been filtered to clearly show the hexagonal structure of graphite. The squarelike quality of the atoms results from a relatively large contact force between the tip and sample. ${ }^{18}$ To maximize sensitivity, the outer portion of the cantilever must be deflected, or biased, by applying a force at the tip. This is undesirable because it is not possible to arbitrarily choose the tip/sample force while maintaining maximal sensitivity. In Fig. 4, the sensitivity was maximized by deflecting the cantilever by roughly $175 \mathrm{~nm}$ (see Fig. 2). As a result, we estimate that the tip/sample force was $0.4 \mu \mathrm{N}$, which is several of orders of magnitude larger than forces typically applied by the AFM. ${ }^{19}$ In future generations of the interdigital cantilever, we plan to bias the deflection of the reference plane in the fabrication process such that maximum sensitivity is achieved without adjusting the deflection of the outer portion of the cantilever.

The authors would like to thank David Bloom, Dan Rugar, Kathryn Wilder, and Digital Instruments for providing valuable information. The primary support for this work came from the Joint Services Electronics Program No. N00014-91-J-1050 of the Office of Naval Research with partial support from NSF. One of the authors (S.R.M.) acknowledges the support of the Urbanek Fellowship and one of the authors (S.C.M.) acknowledges the support of the Leland T. Edwards Fellowship.

${ }^{1}$ G. Binnig, C. F. Quate, and Ch. Gerber, Phys. Rev. Lett. 56, 930 (1986).

${ }^{2}$ G. Meyer and N. M. Amer, Appl. Phys. Lett. 53, 1045 (1988).

${ }^{3}$ S. Alexander, L. Hellemans, O. Marti, J. Schneir, V. Elings, P. K. Hansma, M. Longmire, and J. Gurley, J. Appl. Phys. 65, 164 (1989).

${ }^{4}$ Y. Martin, C. C. Williams, H. K. Wickramasinghe, J. Appl. Phys. 61, 4723 (1987)

${ }^{5}$ R. Erlandsson, G. M. McClelland, C. M. Mate, and S. Chiang, J. Vac. Sci. Technol. A 6, 266 (1988)

${ }^{6}$ D. Rugar, H. J. Mamin, and P. Guethner, Appl. Phys. Lett. 55, 2588 (1989)

${ }^{7}$ C. Schönenberger and S. F. Alvarado, Rev. Sci. Instrum. 60, 3131 (1989); also see D. Anselmetti, Ch. Gerber, B. Michel, H. J. Guntherodt, and H. Rohrer, Rev. Sci. Instrum. 63, 3003 (1992).

${ }^{8}$ M. Tortonese, H. Yamada, R. C. Barrett, and C. F. Quate, in The Proceedings of Transducers ' 91 (IEEE, New York, 1991), IEEE Publication No. 91 CH2817-5, p. 448.

${ }^{9}$ J. K. Gimzewski, Ch. Gerber, E. Meyer, and R. R. Schlittler, Chem. Phys. Lett. 217, 589 (1994).

${ }^{10}$ E. A. Wachter and T. Thundat, Rev. Sci. Instrum. 66, 3662 (1995).

${ }^{11}$ S. S. Lee, L. Y. Lin, and M. C. Wu, Appl. Phys. Lett. 67, 2135 (1995).

${ }^{12}$ O. Solgaard, F. S. A. Sandejas, and D. M. Bloom, Opt. Lett. 17, 688 (1992).

${ }^{13}$ M. Born and E. Wolf, Principles of Optics, 6th ed. (Pergamon, 1980).

${ }^{14}$ Multi-mode Head, Digital Instruments, 520 E. Montecito St, Santa Barbara, California 93103.

${ }^{15}$ The thermal mechanical noise of the cantilever can be estimated by $\sqrt{2 k_{b} T B / \pi Q k f_{0}}$, where $k_{b}$ is the Boltzman factor, $T$ is temperature (300 $\mathrm{K}), Q$ is quality factor $(\sim 100), k$ is the spring constant $(2 \mathrm{~N} / \mathrm{m}), f_{0}$ is the resonant frequency $(50 \mathrm{kHz})$, and $B$ is the bandwidth $(1 \mathrm{kHz})$. This equation yields a value of $0.005 \AA$.

${ }^{16}$ D. Sarid, Scanning Force Microscopy (Oxford University Press, New York, 1991).

${ }^{17}$ The shot noise current is given by $\delta i=\sqrt{2 e R P B}$ where $e$ is the electron charge $\left(1.6 \times 10^{-19} \mathrm{C}\right), R$ is the responsivity of the detector $(\sim 0.5 \mathrm{~A} / \mathrm{W}$ for silicon), $P$ is the laser power $(225 \mu \mathrm{W})$, and $B$ is the bandwidth (1 $\mathrm{kHz})$.

${ }^{18}$ S. Morita, S. Fujisawa, and Y. Sugawara, Surf. Sci. Rep. 23, (1996).

${ }^{19} \mathrm{We}$ estimate the spring constant of our cantilevers to be on the order of 2 $\mathrm{N} / \mathrm{m}$. When displaced by $175 \mathrm{~nm}$, the resulting force is $0.4 \mu \mathrm{N}$. Commercially available $\mathrm{Si}_{3} \mathrm{~N}_{4}$ cantilevers have force constants below $0.01 \mathrm{~N} / \mathrm{m}$ and can be operated with minimal setpoint deflection. 